\title{
trioPhaser: using Mendelian inheritance logic to improve genomic phasing of trios
}

\author{
Dustin B. Miller and Stephen R. Piccolo * (D)
}

\section{*Correspondence:}

stephen_piccolo@byu.edu Department of Biology, Brigham Young University, Provo, UT 84602, USA

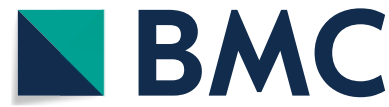

(c) The Author(s), 2021. Open Access This article is licensed under a Creative Commons Attribution 4.0 International License, which permits use, sharing, adaptation, distribution and reproduction in any medium or format, as long as you give appropriate credit to the original author(s) and the source, provide a link to the Creative Commons licence, and indicate if changes were made. The images or other third party material in this article are included in the article's Creative Commons licence, unless indicated otherwise in a credit line to the material. If material is not included in the article's Creative Commons licence and your intended use is not permitted by statutory regulation or exceeds the permitted use, you will need to obtain permission directly from the copyright holder. To view a copy of this licence, visit http:// creativecommons.org/licenses/by/4.0/. The Creative Commons Public Domain Dedication waiver (http://creativecommons.org/publi cdomain/zero/1.0/) applies to the data made available in this article, unless otherwise stated in a credit line to the data.
Results: trioPhaser uses gVCF files from an individual and their parents as initial input, and then outputs a phased VCF file. Input trio data are first phased using Mendelian inheritance logic. Then, the positions that cannot be phased using inheritance information alone are phased by the SHAPEIT4 phasing algorithm. Using whole-genome sequencing data of 52 trios, we show that trioPhaser, on average, increases the total number of phased positions by $21.0 \%$ and $10.5 \%$, respectively, when compared to the number of positions that SHAPEIT4 or Mendelian inheritance logic can phase when either is used alone. In addition, we show that the accuracy of the phased calls output by trioPhaser are similar to linked-read and read-backed phasing.

Conclusion: trioPhaser is a containerized software tool that uses both Mendelian inheritance logic and SHAPEIT4 to phase trios when gVCF files are available. By implementing both phasing methods, more variant positions are phased compared to what either method is able to phase alone.

Keywords: Haplotyping, Phasing, Trios, Genomics, Next-generation sequencing

\section{Background}

When analyzing whole-genome sequence (WGS) data of an individual, the parent of origin for each nucleotide is often unknown [1, 2]. Investigators can identify homozygous-alternate and simple-heterozygous variants, but more complex variants, such as compound-heterozygous variants, are unidentifiable unless the 
genotype data are phased [3, 4]. Phasing (haplotyping) is a process that helps differentiate between maternally and paternally derived nucleotides [1]. Phase can be estimated with computational methods that rely on haplotype evidence from multiple sources; different software programs may support one or more of the following sources: haplotype reference panel, sequence reads, and/or familial genotypes [1, 5-7]. It has been shown that using trio data (data from each parent and the child) as part of the phasing process can improve the number of accurately phased genotypes [1]. In fact, approximately $67-83 \%$ of an individuals' heterozygous sites can be phased using Mendelian inheritance logic [1, 8].

Some computational phasing programs, such as WhatsHap [6] and SHAPEIT2 [9] can take parental genotypes into consideration when making haplotype calls for the child. However, these programs have limitations. For example, WhatsHap requires BAM and VCF files as input [6]. BAM files from WGS data can be over 100 gigabytes in size, thereby requiring much computational storage when many samples are being analyzed [10]. In addition, WhatsHap only outputs phased heterozygous positions, which requires the user to add homozygous alternate positions back into the phased file. SHAPEIT2 can use a single input type or a combination of input types to estimate phase. Input types supported by SHAPEIT2 include VCF files, BAM files, a haplotype reference panel, and parental genotypes [9]. However, SHAPEIT2 does not provide a haplotype reference panel that supports genome build GRCh 38 . Therefore, VCF files that were generated using GRCh38 require conversion to a previous build through a process that may cause hundreds of thousands of variants to be removed, thereby reducing the total number of variants that are available to be phased [11]. A newer phasing method, SHAPEIT4, does not take parental genotype data as direct input into the phasing program, but it does allow pre-phased data to be used as input to increase the accuracy of haplotype calls, and it does support data aligned with GRCh38 [5]. However, SHAPEIT4 does not provide a means to prephase the data, nor does it retain all of the pre-phased data in the final output for variant positions that are not listed in the haplotype reference panel file or that are multi-allelic. Thus, we created trioPhaser, a single-step, containerized application that accepts gVCF files from trio(s) as input, pre-phases the data using Mendelian inheritance logic, and then uses the pre-phased data as input into SHAPEIT4, producing a phased VCF file (Fig. 1).

Our method overcomes some of the limitations that current phasing software presents, without creating a completely new phasing algorithm. We are able to use the information that gVCF files provide to pre-phase the data of the child using Mendelian inheritance as a guide. gVCF files are different from VCF files in that both variant and invariant positions are included as part of the file. This proves beneficial as parental genotype information can be compared to a child's, position for position, in an effort to determine which parent each nucleotide was inherited from. This pre-phase step allows SHAPEIT4 to use a priori information as part of the phasing process and outputs the data in a conventional format with the paternal allele first, followed by the maternal allele. The phased output file generated by SHAPEIT4 excludes a priori positions that are not contained in the haplotype reference panel or that are multi-allelic. 


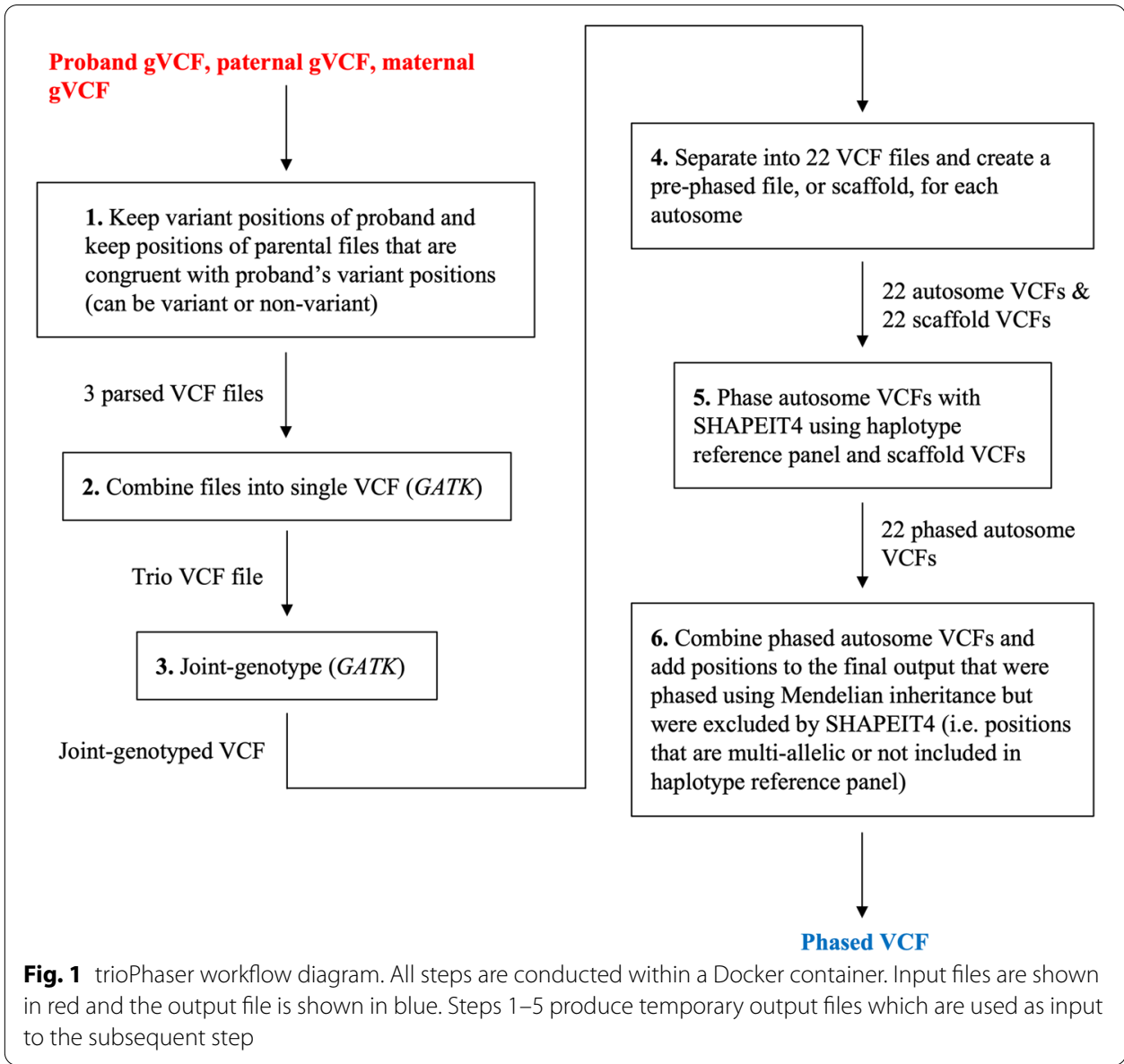

Therefore, after SHAPEIT4 phases the data, trioPhaser adds any Mendelian-phased positions to the final output that were excluded.

\section{Implementation}

\section{Software}

trioPhaser consists of a single Python (https://python.org) script ("trio_phaser.py") that executes within a Docker container [12]. This script provides logic for processing data files and invoking third-party tools including GATK [13] (version 4.0.5.1), bcftools [14] (version 1.9), and SHAPEIT4 [5] (version 4.1.3). These tools are available within the container. Therefore, in order to execute trioPhaser, the user needs only to install the Docker engine and download the Docker image. When multiple cores are available, SHAPEIT4 will phase multiple autosomal chromosomes simultaneously, thereby decreasing run-time.

\section{Inputs}

When a single trio is being phased, trioPhaser has 5 required arguments: (1) gVCF file of the child, (2) gVCF file of the father, (3) gVCF file of the mother, (4) name of the phased output file, and (5) path where haplotype reference files will be saved. 
Alternatively, when multiple trios need to be phased, trioPhaser has 2 required arguments: (1) a TSV file that contains one trio per line where the first, second, and third columns are file paths to the child, father, and mother gVCF file, respectively, and the fourth column is the path to the output file, and (2) path where haplotype reference files will be saved. There are also 3 optional arguments regardless of whether a single trio or multiple trios are phased: (1) the number of CPU cores to use for processing (default $=2),(2)$ which genome build the input files were created with (default $=$ GRCh38, GRCh37 is also supported), and (3) the minimum Phred-scaled quality score a variant position is able to have (default $=30$ ).

\section{Pipeline design}

Although trioPhaser executes with a single Python script, there are six steps performed by the script in order to produce a phased VCF file (Fig. 1). For step 1, the child's gVCF file is used to produce a temporary file that contains all variant positions. In addition, the gVCF files for each parent are used to produce temporary VCF files that contain positions that are congruent with the child's retained variant positions. These positions may be variant or invariant as long as they are found within the newly created file. The purpose of this step is to retain pertinent information and decrease the size of the files. Decreasing file sizes decreases the run-time of subsequent steps.

Step 2 uses GATK's "CombineGVCFs" tool to combine the temporary VCF files from Step 1 into a single file. Then, step 3 uses GATK's "GenotypeGVCFs" tool to joint-genotype the calls. Joint-genotyping can help determine if a poorly called variant is a "true" variant by comparing it to the other samples.

Once genotyped, step 4 separates the file into $22 \mathrm{VCF}$ files and creates a prephased file, or scaffold, for each autosome. To create the scaffold files, Mendelian inheritance logic is used. For positions of the child that can be used to determine which variant came from which parent, the haplotypes of these positions are written to a temporary scaffold file. For example, if the mother is homozygotic for the reference allele (A/A), the father is heterozygotic $(A / G)$, and the child is heterozygotic $(A / G)$, then it can be determined that the reference allele (A) was inherited from the mother and the variant allele $(G)$ was inherited from the father. However, when both parents and the child are heterozygotic (A/G), Mendelian inheritance alone cannot be used to determine which nucleotide came from which parent. SHAPEIT4 uses surrounding pre-phased positions and information from the 1000 Genomes Project [15] haplotype reference panel to phase in such scenarios.

Step 5 uses the autosome VCF files, the scaffold VCF files, and the haplotype reference panel as input into SHAPEIT4 to phase each autosome under default parameters. Only positions that are in the haplotype reference panel and that are bi-allelic are included in the phased VCF files output by SHAPEIT4. Therefore, step 6 uses the scaffold files and the SHAPEIT4-phased chromosome files to create a single VCF file that includes all Mendelian-phased and SHAPEIT4-phased positions for each chromosome. In addition, a separate VCF file is created that only includes phased positions of the child where one of the nucleotides was not identified in either parent. 
These phased positions may be the result of genotype errors or de novo variants. This file is meant to be informative and allow the user to determine how these positions should be used in downstream analyses.

\section{Results}

\section{trioPhaser results for $\mathbf{5 2}$ trios}

Whole-genome sequencing (WGS) data for an Ashkenazim trio and a Han Chinese trio were used to test trioPhaser. Data for these trios were generated by the Genome in a Bottle (GIAB) Consortium [16]. We used GRCh38-aligned BAM files (available through GIAB FTP server) as input into GATK's "HaplotypeCaller" tool to generate gVCF files for each member of each trio. The gVCF files for each trio were then used as input into trioPhaser.

In addition to validating trioPhaser with GIAB data, we ran trioPhaser on 50 trios where the child in each trio had been diagnosed with neuroblastoma. These trios and their associated gVCF files are available through the Gabriella Miller Kids First Data Resource Center [17]. The gVCF files were generated using WGS data.

For the GIAB and 50 neuroblastoma trios, trioPhaser produced a greater number of phased positions than would be provided by using either Mendelian inheritance logic or SHAPEIT4, exclusively (Table 1). Mendelian inheritance logic can be used to phase a greater number of positions than SHAPEIT4 because Mendelian inheritance logic can be applied to multi-allelic positions and does not rely on a haplotype reference panel. However, SHAPEIT4 provides the added benefit of being able to phase positions where all individuals in a trio are heterozygous for the same nucleotides (given that the positions are bi-allelic and are found within the haplotype reference panel). Overall runtime varies for any given trio based on the number of CPUs available, total number of genotyped variants, and variant Phred-scaled quality threshold. For example, average run-time across the 50 neuroblastoma trios was less than the GIAB trios because at a Phred-scaled quality threshold of 30 , there were fewer variants available for phasing than the GIAB trios.

Table 1 trioPhaser results for an Ashkenazim trio, Han Chinese trio, and 50 neuroblastoma trios

\begin{tabular}{llll}
\hline & Ashkenazim trio & Han Chinese trio & 50 neuroblastoma trios \\
\hline Genotyped variants & $5,477,879$ & $5,040,806$ & $\begin{array}{l}5,976,380 \\
\text { sd: } 321,208\end{array}$ \\
Variants phased by trioPhaser & $4,640,241(84.7 \%)$ & $4,490,039(89.1 \%)$ & $\begin{array}{l}4,469,479(75.0 \%) \\
\text { sd: } 171,916\end{array}$ \\
& & & $721,415(16.1 \%)$ \\
Variants phased exclusively using Mende- & $867,181(18.7 \%)$ & $767,992(17.1 \%)$ & sd: 54,522 \\
lian inheritance logic & & & $420,793(9.4 \%)$ \\
Variants phased exclusively by SHAPEIT4 & $458,361(9.9 \%)$ & $417,138(9.3 \%)$ & sd: 15,414 \\
Run-time (22 CPUs used) & $5.8 \mathrm{~h}$ & $5.4 \mathrm{~h}$ & $4.4 \mathrm{~h}$ \\
\hline
\end{tabular}

trioPhaser uses Mendelian inheritance in conjunction with SHAPEIT4. This hybrid approach produced a greater number of phased positions than was provided using either method exclusively. Results for the 50 neuroblastoma trios were averaged $\mathrm{sd}=$ Standard deviation 


\section{Comparison of trioPhaser to linked-read phasing technology}

In addition to BAM files, GIAB provides phased VCF files for each member of the Ashkenazim trio and Han Chinese trio. These data had been phased using linked-read technology (10X Genomics) which is a laboratory-based phasing method that randomly partitions small amounts of DNA to decrease the chance of a single partition having DNA from the same genomic position $[1,18]$. This laboratory-based phasing method has been shown to be one of the most accurate [1]. Therefore, we used these trios as our "gold standard" to evaluate how similar the phased results of trioPhaser were to the linked-read technology results.

trioPhaser produced similar phased calls as linked-read phasing technology and is a suitable alternative for phasing trios. For the Ashkenazim trio, trioPhaser and 10X Genomics had 3,999,945 variants that were congruent (those that had the same position, reference allele, and alternate allele). Of the congruent variants, 3,952,525 (98.8\%) were phased identically. For the Han Chinese trio, 4,000,682 variants were congruent between the 10X-phased and trioPhaser-phased data; of these variants, 3,900,447 (97.5\%) were phased identically.

\section{Comparison of trioPhaser to WhatsHap}

WhatsHap is a read-based phasing method that uses sequencing reads to reconstruct haplotypes [6]. This phasing method requires BAM file(s) and a VCF file as inputs. Incorporating sequencing reads as part of the phasing process can increase the overall accuracy of phase results [1]. Therefore, we phased the Ashkenazim and Han Chinese trios using WhatsHap to compare the phasing output of read-backed phasing to trioPhaser. Before phasing with WhatsHap, we used GATK's "CombineGVCFs" tool to combine the gVCF files we previously created for the Ashkenazim trio and Han Chinese trio, and then joint-genotyped each combined trio using GATK's "GenotypeGVCFs" tool. This joint-genotyped file for each trio and the GRCh38-aligned BAM files for each member of each trio were used as input into WhatsHap. The phased VCF output by WhatsHap only includes heterozygous positions. Therefore, we only compared heterozygous positions between trioPhaser and WhatsHap.

trioPhaser produced similar phased calls on heterozygous positions as WhatsHap and is a suitable phasing alternative. For the Ashkenazim trio, trioPhaser and WhatsHap had $2,275,546$ phased heterozygous variants that were congruent. Of the congruent variants, 2,204,240 (96.9\%) were phased identically. 1,959,580 (88.9\%) of the congruent positions could be phased using Mendelian inheritance logic and 100\% percent of these positions were phased identically between trioPhaser and WhatsHap. WhatsHap took $26.6 \mathrm{~h}$ to phase compared to trioPhaser, which took 5.8 h. For the Han Chinese trio, 2,070,536 phased heterozygous variants were congruent between trioPhaser and WhatsHap and of these variants, 1,997,276 (96.5\%) were phased identically. 1,777,588 (89.0\%) of the congruent positions could be phased using Mendelian inheritance logic, and 100\% percent of these positions were phased identically between trioPhaser and WhatsHap. WhatsHap took $26.6 \mathrm{~h}$ to phase compared to trioPhaser, which took $5.4 \mathrm{~h}$.

The source code and a detailed document explaining where data was downloaded from, how it was processed, and how to run trioPhaser is available at https://github. com/dmiller903/trioPhaser. 


\section{Conclusion}

trioPhaser is a containerized phasing tool that implements Mendelian inheritance logic and SHAPEIT4 to phase trios. Using both phasing methods produces a greater overall number of phased variants than would be output when using either method alone. In fact, on average, trioPhaser increased the total number of phased variants by $21.0 \%$ and $10.5 \%$, respectively, when compared to what SHAPEIT4 or Mendelian inheritance logic were able to phase alone. We show that, on average, $98.2 \%$ of the congruent 10X-phased haplotype calls, and $96.7 \%$ of the congruent WhatsHap haplotype calls are the same as trioPhaser. trioPhaser is a suitable phasing alternative for trios when gVCF files are available or can be generated.

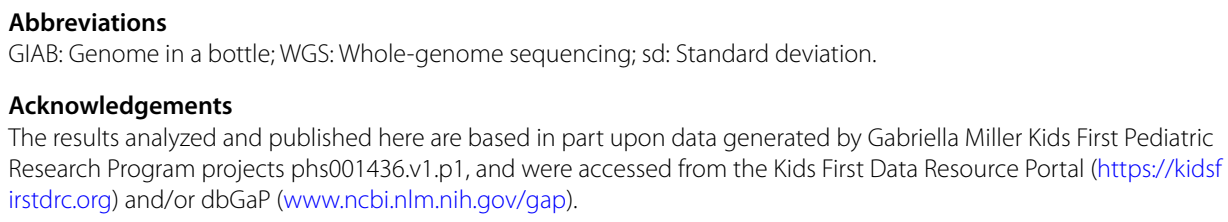

Authors' contributions

DBM developed trioPhaser, wrote and tested the code, and prepared the manuscript. SRP provided insight and edited the manuscript. Both authors read and approved the final manuscript.

\section{Funding}

Not applicable

\section{Availability of data and materials}

The Genome in a Bottle data underlying this article are available at ftp://ftp-trace.ncbi.nlm.nih.gov/ReferenceSamples/ giab/data/AshkenazimTrio/analysis/10XGenomics_ChromiumGenome_LongRanger2.0_06202016/. The Gabriella Miller Kids First data underlying this article cannot be shared publicly due to access restrictions. However, access for these data can be requested through dbGap at https://www.ncbi.nlm.nih.gov/projects/gap/cgi-bin/study.cgi?study_id=phs00 1436.v1.p1.

\section{Availability and requirements}

Project name: trioPhaser.

Project home page: https://github.com/dmiller903/trioPhaser.

Project source code: https://github.com/dmiller903/trioPhaser.

Operating system: Linux, Windows, or MacOS.

Programming language: Python3.

Other requirements: trioPhaser requires that the Docker engine be installed.

License: MIT.

\section{Declarations}

Ethics approval and consent to participate

Not applicable.

\section{Consent for publication}

Not applicable.

\section{Competing interests}

The authors declare that they have no competing interests.

Received: 18 June 2021 Accepted: 8 November 2021

Published online: 22 November 2021

\section{References}

1. Choi Y, Chan AP, Kirkness E, Telenti A, Schork NJ. Comparison of phasing strategies for whole human genomes. PLoS Genet. 2018;14:e1007308.

2. Snyder MW, Adey A, Kitzman JO, Shendure J. Haplotype-resolved genome sequencing: experimental methods and applications. Nat Rev Genet. 2015;16:344-58.

3. Miller D, Piccolo S. Compound heterozygous variants in pediatric cancers: a systematic review. Front Genet. 2020;11:493. 
4. Miller DB, Piccolo SR. a survey of compound heterozygous variants in pediatric cancers and structural birth defects. Front Genet. 2021;12:363.

5. Delaneau O, Zagury J-F, Robinson MR, Marchini JL, Dermitzakis ET. Accurate, scalable and integrative haplotype estimation. Nat Commun. 2019;10:5436.

6. Martin M, Patterson M, Garg S, Fischer SO, Pisanti N, Klau GW, Schöenhuth A, Marschall T. WhatsHap: fast and accurate read-based phasing 2016. bioRxiv:085050.

7. Garg S. Computational methods for chromosome-scale haplotype reconstruction. Genome Biol. 2021;22:101.

8. Roach JC, Glusman G, Hubley R, Montsaroff SZ, Holloway AK, Mauldin DE, Srivastava D, Garg V, Pollard KS, Galas DJ, Hood L, Smit AFA. Chromosomal haplotypes by genetic phasing of human families. Am J Hum Genet. 2011;89:382-97.

9. Delaneau O, Howie B, Cox AJ, Zagury J-F, Marchini J. Haplotype estimation using sequencing reads. Am J Hum Genet. 2013;93:687-96.

10. Greenfield D, Wittorff V, Hultner M. The importance of data compression in the field of genomics. IEEE Pulse. 2019;10:20-3

11. Miller DB, Piccolo SR. CompoundHetVIP: compound heterozygous variant identification pipeline. F1000Res. 2020;9:1211.

12. Boettiger C. An introduction to Docker for reproducible research. Oper Syst Rev. 2015;49:71-9.

13. GATK/Tool Documentation Index. https://software.broadinstitute.org/gatk/documentation/tooldocs/3.8-0/org_ broadinstitute_gatk_tools_walkers_phasing_ReadBackedPhasing.php.

14. Li H, Handsaker B, Wysoker A, Fennell T, Ruan J, Homer N, Marth G, Abecasis G, Durbin R, 1000 Genome Project Data Processing Subgroup. The sequence alignment/map format and SAMtools. Bioinformatics 2009; 25:2078-2079.

15. 1000 Genomes Project Consortium, Auton A, Brooks LD, Durbin RM, Garrison EP, Kang HM, Korbel JO, Marchini JL, McCarthy S, McVean GA, Abecasis GR. A global reference for human genetic variation. Nature 2015; 526:68-74.

16. Zook JM, McDaniel J, Olson ND, Wagner J, Parikh H, Heaton H, Irvine SA, Trigg L, Truty R, McLean CY, De La Vega FM, Xiao C, Sherry S, Salit M. An open resource for accurately benchmarking small variant and reference calls. Nat Biotechnol. 2019;37:561-6.

17. Heath AP, Taylor DM, Zhu Y, Raman P, Lilly J, Storm P, Waanders AJ, Ferretti V, Yung C, Mattioni M, Davis-Dusenbery B, Flamig ZL, Grossman R, Volchenboum SL, Mueller S, Nazarian J, Vasilevsky N, Haendel MA, Resnick A. Abstract 2464: Gabriella Miller kids first data resource center: harmonizing clinical and genomic data to support childhood cancer and structural birth defect research. Cancer Res. 2019;79:2464-2464.

18. Zheng GXY, Lau BT, Schnall-Levin M, Jarosz M, Bell JM, Hindson CM, Kyriazopoulou-Panagiotopoulou S, Masquelier DA, Merrill L, Terry JM, Mudivarti PA, Wyatt PW, Bharadwaj R, Makarewicz AJ, Li Y, Belgrader P, Price AD, Lowe AJ, Marks P, Vurens GM, Hardenbol P, Montesclaros L, Luo M, Greenfield L, Wong A, Birch DE, Short SW, Bjornson KP, Patel P, Hopmans ES, Wood C, Kaur S, Lockwood GK, Stafford D, Delaney JP, Wu I, Ordonez HS, Grimes SM, Greer S, Lee JY, Belhocine K, Giorda KM, Heaton WH, McDermott GP, Bent ZW, Meschi F, Kondov NO, Wilson R, Bernate JA, Gauby S, Kindwall A, Bermejo C, Fehr AN, Chan A, Saxonov S, Ness KD, Hindson BJ, Ji HP. Haplotyping germline and cancer genomes with high-throughput linked-read sequencing. Nat Biotechnol. 2016;34:303-11.

\section{Publisher's Note}

Springer Nature remains neutral with regard to jurisdictional claims in published maps and institutional affiliations.

- fast, convenient online submission

- thorough peer review by experienced researchers in your field

- rapid publication on acceptance

- support for research data, including large and complex data types

- gold Open Access which fosters wider collaboration and increased citations

- maximum visibility for your research: over 100M website views per year

At BMC, research is always in progress.

Learn more biomedcentral.com/submissions 